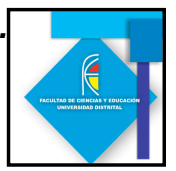

\title{
ENSEÑANZA DE LA CIENCIAS EN AULAS INCLUSIVAS.
}

\section{SCIENCE TEACHING IN INCLUSIVE CLASSROOMS.}

\author{
María Andrea Hernández Ballesteros ${ }^{1}$ \\ Rusby Malagon ${ }^{2}$ \\ Germán Bautista ${ }^{3}$
}

\begin{abstract}
Resumen
Esta investigación se realiza con el fin de reflexionar y de aportar al proceso de enseñanza- aprendizaje de aquellos estudiantes con déficit cognitivo (DC) incluidos en las aulas regulares. La propuesta nace a partir de la problemática que se evidenció desde la práctica docente, donde se logró visualizar que la inclusión de estas poblaciones al aula regular resulta ser una tarea que presenta muchas dificultades a los docentes que la enfrentan por las condiciones cognitivas que los estudiantes poseen, como por ejemplo, abstraer ideas sencillas. Las estrategias que usualmente se trabajan para que esta población acceda al conocimiento de las ciencias que demandan acciones de pensamiento de un alto nivel de abstracción en general no son las más adecuadas. La investigación pretende diseñar y aplicar una estrategia de aula que estimule el desarrollo de habilidades de pensamiento a partir del estudio de las manifestaciones de la energía. Estrategia que favorecerá la caracterización de los obstáculos y fortalezas presentes en los procesos cognitivos en el aprendizaje de la física con estos estudiantes, así mismo, favorecerá la identificación de algunas de las acciones didácticas de mayor pertinencia para la enseñanza de las ciencias con estas poblaciones con necesidades educativas especiales particulares (NEEP).
\end{abstract}

Palabras Clave: Enseñanza, Inclusión, Déficit Cognitivo, didáctica, ciencias, transformación de la energía.

\begin{abstract} implement a classroom strategy that encourages development of thinking skills by the $s$

\footnotetext{
${ }^{1}$ Estudiante de Licenciatura de física, Universidad Pedagógica Nacional, Mahb.1986@gmail.com

2 Profesor de la Universidad Pedagógica Nacional

${ }^{3}$ Profesor de la Universidad Pedagógica Nacional
}

This research was performed in order to contribute to the teaching- learning processes of students with cognitive deficits (DC) included in the regulars classrooms. This proposal stem from the problems that became evident from the teaching practice. The inclusion of these populations to the regular classroom turns out to be a task that presents many challenges to the teacher given the special cognitive conditions these students have. The usual strategies followed in order to guide the students to access scientific knowledge that requires high level of abstraction are not adequated. This research aims to design and 


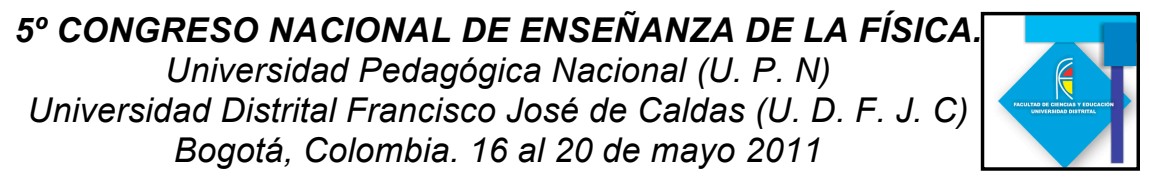

tudy of the manifestations of energy in several physical processes. This strategy is aimed to find some characterization of some obstacles in cognitive processes of physics learning as well. Likewise to identify some of the teaching activities of most relevance to science teaching.

Keywords: Education, Inclusion, Cognitive deficits, didactic, science, energy transformation.

\section{Reconociendo la población}

Una de las preocupaciones que ha tenido la línea de investigación "Construcción del Conocimiento Científico desde las Perspectivas de los Enfoques Didácticos" del Departamento de Física de la Universidad Pedagógica Nacional es la inclusión al aula regular. Consideramos que la labor del docente de ciencias puede llegar a ser tan fructífera en la medida en que el docente conozca y reconozca sus estudiantes pero ¿Qué sucede cuando el docente en ese reconocimiento descubre que en su aula de clase se encuentra un o unos estudiantes con alguna limitación? Es compleja esta situación porque nuestra formación es simplemente en alguna disciplina de la ciencias, nosotros los docentes de ciencias en nuestro proceso de formación solo tenemos una manera enseñar. En este orden de ideas se realizó el proyecto titulado Aprendizaje Mediado Con Estudiantes Que Presentan Déficit Cognitivo En Aulas Inclusivas: una experiencia didáctica para aproximar a la comprensión de la transformación de la energía.

El cual se lleva a cabo en la sede central de la Institución Educativa José Martí IED ubicada en la localidad Rafael Uribe Uribe (Localidad 18 de Bogotá D.C. Colombia), que cuenta con cinco sedes.

Esta Institución educativa posee aulas inclusivas con estudiantes con déficit cognitivo. El presente proyecto investigativo se realizó con nueve estudiantes en total, seis tienen déficit cognitivo los restantes son regulares. Por otro lado todos poseen habilidades de independencia y autonomía fisiológicas es decir control de esfínteres, comen por sí mismos, se desplazan de un lugar otro por si solos (excepto un estudiante con (DC) es el único que es minusválido y su desplazamiento es a través de una silla de ruedas.) Asimismo pueden convivir con los demás en un mismo lugar sin que presenten problemas de agresividad, también se detecto que los estudiantes con déficit cognitivo en su totalidad presentan problemas de concentración, inseguridad, tienen problemas para abstraer ideas centrales. Por supuesto detectamos esto a través de la aplicación de unos instrumentos diagnósticos.

Nota: Todos los estudiantes con déficit cognitivo presentan deficiencia de leve a moderada, es decir, pueden ser escolarizados.

También es importante para el desarrollo de este proyecto tener en cuenta los problemas relacionados con la etapa de la adolescencia ya que estos estudiantes presentan dos edades al tiempo; su edad cronológica que es la que hace que ellos quieran ser tratados como adultos, pero su edad mental es la de un niño hace que tengan que ser tratados de 


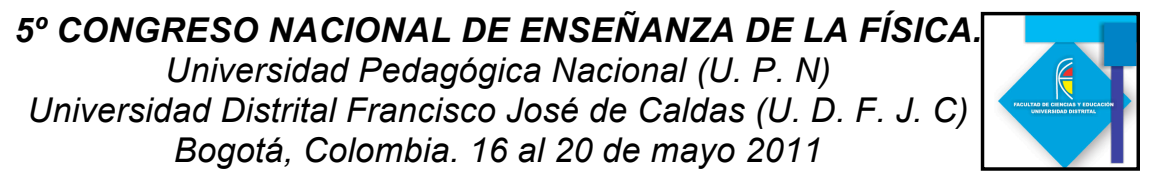

esta manera. Esto genera que por una parte se sientan ridiculizados debido que reciben un trato como de niños.

Por otro lado se realizó una búsqueda teórica con la cual se fundamenta este proyecto, de esta manera se indaga sobre la teoría de la Modificabilidad cognitiva por el Dr. Reuven Feuerstein. Se hace uso de algunos parámetros de esta teoría para la creación de la propuesta didáctica para la enseñanza- aprendizaje con estudiantes con déficit cognitivo.

Por otro lado se considera que el fortalecimiento de las habilidades del pensamiento permitirá a cualquier estudiante aprender de forma significativa, además de generar autonomía en su aprendizaje.

La documentación en textos frente a la transformación de la terminología en relación a la integración pro inclusión y las implicaciones que tuvo el cambio de termino dentro de la escuela.

Se realizó ante todo una contextualización histórica de el concepto de transformación de la energía, conservación de la energía, fuentes de energía. Aunque en el momento de la aplicación de la estrategia se trata solo la transformación de la energía como se evidenciará más adelante.

\section{La propuesta:}

Se diseñó una posible ruta didáctica para reconocer y fortalecer las habilidades del pensamiento de los estudiantes regulares y con déficit cognitivo a propósito de la inclusión en Colombia. Todo esto a través del estudio de la transformación de la energía.

Se establece tres intervenciones, cada una con una serie de momentos que tienen una duración de treinta a sesenta minutos, con mayor duración los estudiantes no prestarán la atención que requiere las actividades.

Se considera que estas intervenciones tengan una duración de un mes completo (tiempo promedio en el que se trabajaría un tema cualquiera en un aula de clase). A continuación se presenta una tabla con un esquema general de las actividades trabajadas con los estudiantes.

\begin{tabular}{|c|c|c|}
\hline $\begin{array}{l}\text { Intervención } \mathbf{1} \\
\text { Reconociendo el aula inclusiva }\end{array}$ & $\begin{array}{l}\text { ción } \mathbf{2} \\
\text { ando ideas! }\end{array}$ & $\mathrm{a!}$ \\
\hline $\begin{array}{l}\text { Momento 1 } \\
\text { Realizar valoración de algunos } \\
\text { aspectos sociales del grupo de } \\
\text { estudiantes regulares y con } \\
\text { deficiencia intelectual }\end{array}$ & $\begin{array}{l}\text { Momento } 1 \\
\text { Permitir que los estudiantes } \\
\text { relacionen los conceptos de } \\
\text { conservación de una cantidad, a } \\
\text { partir del juego monopolio a gran } \\
\text { escala (los niños serán las fichas). }\end{array}$ & $\begin{array}{l}\text { Momento } 1 \\
\text { Formalizar los conceptos de } \\
\text { conservación, transformación de la } \\
\text { energía. } \\
\text { Momento } 2\end{array}$ \\
\hline $\begin{array}{l}\text { Realizar valoración de algunos } \\
\text { aspectos cognitivos (habilidades } \\
\text { del pensamiento) del grupo de } \\
\text { estudiantes regulares y con } \\
\text { deficiencia intelectual. A partir del } \\
\text { de la aplicación de unas sencillas } \\
\text { actividades que permitan identificar }\end{array}$ & $\begin{array}{l}\text { Momento } 2 \\
\text { Vincular a los estudiantes con los } \\
\text { conceptos de conservación y } \\
\text { transformación de una cantidad a } \\
\text { partir de la construcción de figuras } \\
\text { utilizando el tangram. }\end{array}$ & $\begin{array}{l}\text { A partir de experiencias sencillas } \\
\text { evidenciar de la transformación de } \\
\text { la energía. } \\
\text { Momento } 3 \\
\text { Identificar cuáles fueron las } \\
\text { compresiones y la significación que } \\
\text { realizaron todos los estudiantes a }\end{array}$ \\
\hline
\end{tabular}




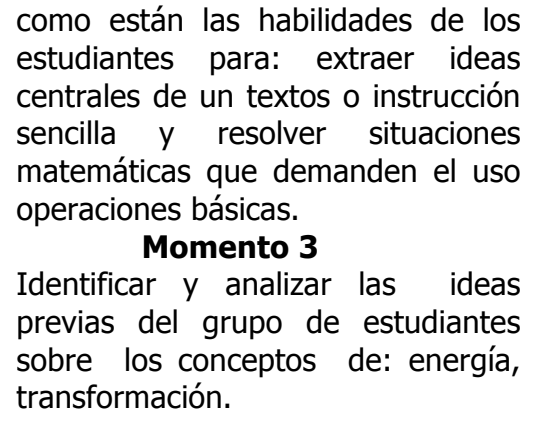

Tabla 1 Esquema general de la propuesta didáctica

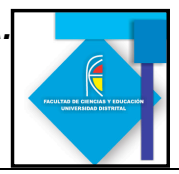

partir del tema tratado.

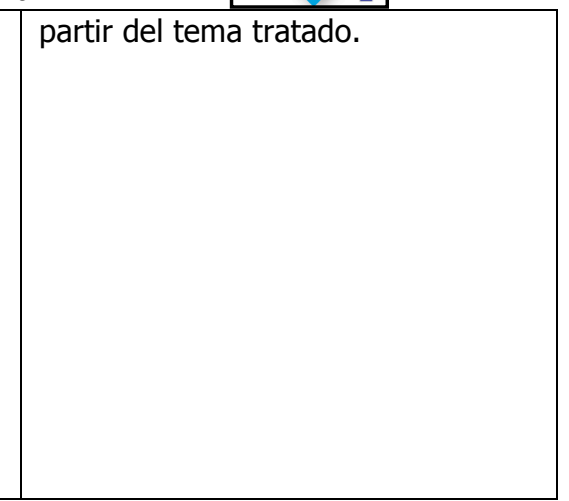

Análisis:

\section{Intervención 1 Reconociendo el aula inclusiva}

Momento 1: Identificación de aspectos sociales

Se realizó una entrevista estructurada dirigida a todos los estudiantes que participaran de la implementación de la estrategia, con el fin de identificar algunos aspectos sociofamiliares. Se puede concluir que los estudiantes en su mayoría no tienen padres y se encuentran en entorno bastante agresividad.

\section{Momento 2: Identificación de aspectos cognitivos}

Se desarrolló con los estudiantes actividades sencillas que tiene el propósito de identificar cómo están sus habilidades para extraer las ideas centrales de un texto y expresar su pensamiento, y de Identificar cómo está sus habilidades para resolver situaciones sencillas haciendo uso de las operaciones básicas. En estas actividades se concluyó que los estudiantes con (DC) tienen serios problemas para abstraer ideas centrales, relatar historias etc. En cuanto a las operaciones básicas se les dificulta asociar problemas de la vida cotidiana con las matemáticas y sus operaciones básicas.

\section{Momento 3 ¿Qué piensas?: Reconociendo ideas previas}

Se proyectó un video sobre transformación: "Gokú se transforma en súper sayayin fase $3^{\prime \prime}$ y a partir del mismo se formularon algunas preguntas que pretendian identificar la idea que tienen los estudiantes acerca del concepto de transformación. Se identificó la dificultad que tienen los estudiantes con (DC) para asociar la transformación a la vida real.

\section{Intervención 2 iFormando Ideas!}

\section{Momento 1 iQuien tiene más!}

Se realizó un juego llamado monopolio, donde los estudiantes son las fichas del juego. Se le entregó a cada estudiante un dinero por valor de $\$ 300.000$ y se les indicó como recorrer el juego. Se identificó como los estudiantes con (DC) presentan pereza para trabajar ya que por su condición no se exigen a sí mismos. Ellos suponen que uno (Docente) siempre les va ayudar

\section{Momento 2 iorganizando las ideas!}




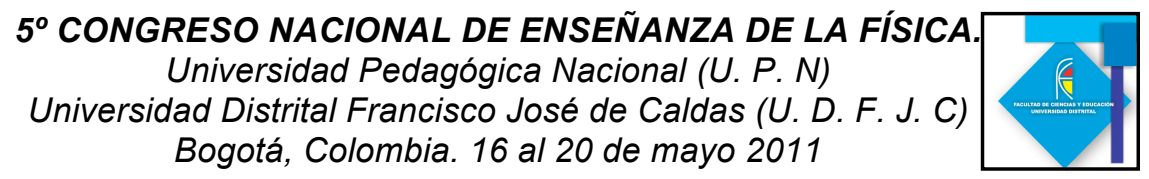

Se le estregó a cada estudiante un tablero de tangram con una

cantidad de siete fichas y una hoja con 5 figuras diferentes, se esperó que los estudiantes con déficit cognitivo logren construir con el tangram las figuras indicadas (de igual manera los niños regulares). De manera simultánea se formularon preguntas para observar como los estudiantes relacionan el ejercicio que están realizando con el concepto de conservación que veníamos trabajando de sesiones anteriores. En general a todo los estudiantes les costó realizar hallar la relación entre la organización de fichas y la transformación.

\section{Intervención 3 iAsí funciona!}

\section{Momento 1iEs así!}

Se les proyectó videos relacionados con la energía y sus transformaciones para que fueran realizando sus propias relaciones sobre los conceptos anteriormente presentados.Despues a partir de preguntas se va orientando a los estudiantes a su propia construcción frente al tema.Relacionaron a partir del video las actividades anteriores, pero no lo relacionan con la vida cotidiana.

\section{Momento 2 iAsí funciona!}

Se les muestra a partir del lanzamiento del balón de bascketball como se transforma la energía y además un dipolo en el cual ellos tienen que realizar un esfuerzo para lograr movimiento del mismo y poder transferir energía a un led. Se motivaron a partir de las experiencias realizadas.

\section{Momento 3 iEntendimos que!}

Para este momento se realizó una mesa redonda en donde la idea es que todos participarán, es así que a partir de preguntas los estudiantes responderán, dándole una significación propia a lo que quieren dar a entender, como existen muchos casos en donde los estudiantes son tímidos, antes de realizar la mesa redonda cada uno tendrá tiempo para responder las preguntas en una hoja y lo que se hace en la mesa redonda es socializar las respuestas a esas preguntas. Aunque se obtuvo construcciones significativas con los estudiantes regulares en su mayoría los estudiantes con (DC) se aproximaron a la transformación de la energía.

\section{Referencias Bibliográficas}

Doré, R y Wagner, S y Brunet, J.P (2002). Integración Escolar. México: PEARSON EDUCACION.

Alinovi, A. (2007). Historia de la energía. Buenos Aires, Argentina: Capital intelectual S.A.

Barrios S y Cortes J. (2002). La enseñanza de las ciencias naturales con apoyo de medios de alta y baja tecnología. Con retardo Mental.

SEARS ZEMANSKY. (2004). Energía Potencial y Conservación de la energía. Undécima edición. Volumen 1. PEARSON Educación.2004 